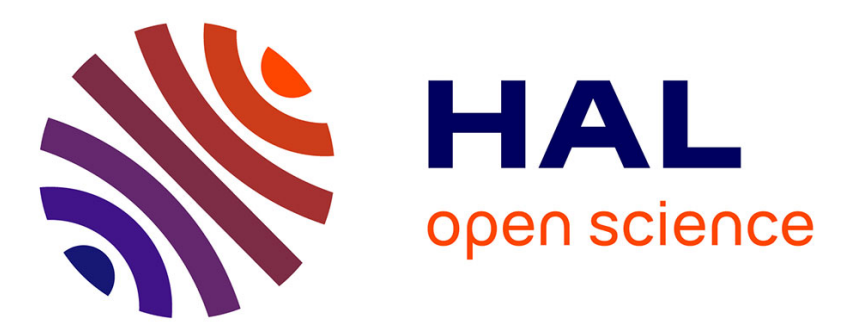

\title{
Mechanism of superexchange Interatomic Coulombic decay in rare gas clusters
}

Petra Votavová, Tsveta Miteva, Selma Engin, Sévan Kazandjian, Přemysl

Kolorenč, Nicolas Sisourat

\section{- To cite this version:}

Petra Votavová, Tsveta Miteva, Selma Engin, Sévan Kazandjian, Přemysl Kolorenč, et al.. Mechanism of superexchange Interatomic Coulombic decay in rare gas clusters. Physical Review A, 2019, 100 (2), pp.022706. 10.1103/PhysRevA.100.022706 . hal-02294413

\section{HAL Id: hal-02294413 \\ https://hal.sorbonne-universite.fr/hal-02294413}

Submitted on 23 Sep 2019

HAL is a multi-disciplinary open access archive for the deposit and dissemination of scientific research documents, whether they are published or not. The documents may come from teaching and research institutions in France or abroad, or from public or private research centers.
L'archive ouverte pluridisciplinaire HAL, est destinée au dépôt et à la diffusion de documents scientifiques de niveau recherche, publiés ou non, émanant des établissements d'enseignement et de recherche français ou étrangers, des laboratoires publics ou privés. 


\title{
Mechanism of superexchange Interatomic Coulombic decay in rare gas clusters
}

\author{
Petra Votavová, ${ }^{1}$ Tsveta Miteva, ${ }^{2}$ Selma Engin, ${ }^{2}$ Sévan \\ Kazandjian, ${ }^{2}$ Přemysl Kolorenč ${ }^{1}$ and Nicolas Sisourat ${ }^{2, *}$ \\ ${ }^{1}$ Charles University, Faculty of Mathematics and Physics, \\ Institute of Theoretical Physics, V Holešovičkách 2, 18000 Prague, Czech Republic \\ ${ }^{2}$ Sorbonne Université, CNRS, Laboratoire de Chimie Physique - Matière et Rayonnement, \\ F-75005 Paris, France
}

(Dated: July 31, 2019)

\begin{abstract}
Interatomic Coulombic Decay (ICD) is an ultrafast energy transfer process. Via ICD, an excited atom can transfer its excess energy to a neighboring atom which is thus ionized. On the example of NeHeNe cluster, we recently reported [Phys. Rev. Lett. 119, 083403 (2017)] that the total ICD widths are substantially enhanced in the presence of an ICD inactive atom. The enhancement occurs due to the coupling of the resonance state to intermediate virtual states of the bridge atom - a mechanism named superexchange ICD. In this follow-up work, we analyze the partial ICD widths in NeHeNe cluster and show that only some channels are affected by the superexchange ICD process. Furthermore, we consider superexchange ICD in NeHeAr. We show that in this system the enhancement is still present but the energy transfer mediated by the superexchange mechanism is less efficient than in NeHeNe owing to the different ionization potentials of Ar and Ne. The behavior of the computed ICD widths is explained with a simple model based on first-order perturbation theory and a Hartree-Fock-like description of the states.
\end{abstract}

\footnotetext{
* nicolas.sisourat@sorbonne-universite.fr
} 


\section{INTRODUCTION}

Interatomic Coulombic Decay (ICD) is an ultrafast energy transfer process between two weakly bound systems [1-3]. In ICD, an excited donor partner has enough excess energy to ionize an acceptor one. The most typical example is that of a neon dimer after 2s ionization of one neon atom within the dimer. This excited neon ion is the donor atom and relaxes within $150 \mathrm{fs}[4,5]$ by transferring its excess energy to the neutral neighboring neon atom (i.e. the acceptor), which is thus ionized.

ICD is a general effect which has been observed in many diverse situations [6-9]. Its mechanism has been discussed in [10]. In the latter reference, the authors showed that when the donor-acceptor distance $\mathrm{R}$ is large the energy transfer can be pictured as an exchange of a virtual photon: the donor emits a virtual photon which is absorbed by the acceptor. In such case, the ICD rates behave as $\mathrm{R}^{-6}$. At shorter interatomic distances, the orbitals of the donor and acceptor systems overlap and the ICD rates are much larger than predicted by the virtual photon mechanism.

Recently, we have shown that another mechanism is possible when the donor and acceptor systems are separated by a bridge atom. On the example of neon-helium-neon trimer, we have shown that ICD between the two neon atoms after 2 s ionization of one neon atom exhibits total widths that are substantially enhanced in the presence of a helium atom compared to the isolated neon dimer [11]. Furthermore, we have shown that this novel mechanism, so-called superexchange ICD, is mediated by the presence of energetically close intermediate configurations. Note also that a virtual photon approximation was recently derived for three-body ICD processes [12].

In the following, we analyze both the total and partial ICD widths of neon-heliumneon and neon-helium-argon trimers. It is shown that, owing to symmetry reasons, only particular channels are enhanced by the superexchange mechanism. We also demonstrate that because of the difference in ionization potential between neon and argon, the superexchange contribution is significantly reduced in the neon-helium-argon system compared to the neon-helium-neon trimer. Furthermore, we present in detail a model based on first-order perturbation theory which provides insights into the superexchange mechanism.

The outline of the article is the following: in section II, we describe the theoretical methods and computational details employed to calculate the total and partial ICD widths. 
Furthermore, a model based on first-order perturbation theory, which helps the interpretation of the ab initio results is detailed. In section III we present and discuss the results for the neon-helium-neon and neon-helium-argon trimers. The article ends with the conclusions of this work. Atomic units are used throughout unless stated otherwise.

\section{METHODS AND COMPUTATIONAL DETAILS}

\section{A. Fano-Configuration Interaction method}

We employed the Fano-Configuration Interaction (Fano-CI) method [13] to compute the total and partial widths of neon-helium-neon and neon-helium-argon trimer after $2 \mathrm{~s}$ ionization of one neon atom. The method was presented in detail in [13], here we outline it briefly.

In Fano theory [14, 15], a resonance is described as a discrete state $\Phi$, embedded in and coupled to one or several continua. The ICD widths are then given by the coupling between the discrete state and the continuum states $\left|\chi_{\beta, \epsilon_{\beta}}\right\rangle$

$$
\Gamma=\sum_{\beta} \Gamma_{\beta}=2 \pi \sum_{\beta}\left|\left\langle\Phi\left|\hat{H}-E_{r}\right| \chi_{\beta, \epsilon_{\beta}}\right\rangle\right|^{2},
$$

where $\Gamma$ is the total width and $\Gamma_{\beta}$ the partial width corresponding to the decay channel $\beta$. The kinetic energy of the ICD electron for a given channel $\beta$ is $\epsilon_{\beta}$ and $E_{r}$ is the energy of the inner-valence ionized state. We use a simple CI scheme to describe both parts: the discrete part is a one-hole $(1 \mathrm{~h})$ configuration where an electron is removed from an innervalence orbital of the Hartree-Fock (HF) determinant of the neutral system. The continuum part is obtained by performing CI calculations where the ICD electron is approximated by the Hartree-Fock virtual orbitals. A CI calculation is performed for each virtual orbital separately, which leads to small sized matrices to be diagonalized. Since the virtual orbitals do not have the proper boundary conditions, a Stieltjes imaging technique is employed to recover the correct widths from the approximated ones [16-18]. Similarly to Ref. [19], we computed the decay width as the average over a range of Stieltjes orders, for which it does not vary substantially.

We used restricted Hartree-Fock molecular orbitals optimized for each system. The aug-cc-pVTZ basis set [20] augmented with [7s,7p,7d] diffuse functions of the KaufmannBaumeister-Jungen (KBJ) type [21] functions was employed on all atoms in the case of the 
NeHeNe trimer and NeNe dimer. The aug-cc-pVQZ basis set [20] augmented with [6s,6p,6d] KBJ functions was used in the case of NeHeAr and NeAr. In order to compute the ICD widths for the trimers and dimers with the same basis sets, a ghost atom $\mathrm{X}$ was placed at the position of He for the NeNe and NeAr calculations. Additionally, [3s,10p,3d] diffuse KBJ functions were added at the mid-points between Ne-He, and He-Ar in the case of NeHeAr, and Ne-X and X-Ar in the case of NeXAr. A larger basis set for NeAr and NeHeAr was thus employed in order to ensure convergence of the results. We attribute the need for a larger basis set for these systems to the higher ICD electron energy.

Within the framework of the Fano-CI method, the partial width $\Gamma_{\beta}$ to a given decay channel can be expressed as

$$
\Gamma_{\beta}=2 \pi\left|\left\langle\Phi\left|\hat{H}-E_{r}\right| \Phi_{\beta}^{2 \mathrm{~h}} k_{\beta}\right\rangle\right|^{2}
$$

where we represent the decay channel as a product of a doubly-ionized CI state $\Phi_{\beta}^{2 h}$ and an electron excited to a virtual orbital $k$. To calculate these quantities, we assume that

the pseudo-continuum final states $\tilde{\chi}_{q}^{a}$ computed using the Fano-CI method form a complete basis. We expand the $\Phi_{\beta}^{2 \mathrm{~h}} k_{\beta}$ wave function in this basis

$$
\Gamma_{\beta}=2 \pi\left|\sum_{a q}\left\langle\Phi\left|\hat{H}-E_{r}\right| \tilde{\chi}_{q}^{a}\right\rangle\left\langle\tilde{\chi}_{q}^{a} \mid \Phi_{\beta}^{2 h} k_{\beta}\right\rangle\right|^{2}
$$

Each Fano-CI state $\tilde{\chi}_{q}^{a}$ is represented as a linear combination of $2 \mathrm{~h} 1 \mathrm{p}$-configurations (singlyionized excited Slater determinants), we can thus re-write the above equation as

$$
\Gamma_{\beta}=2 \pi\left|\sum_{a q}\left(\mathbf{T d}^{a}\right)_{\beta q}\left\langle\Phi\left|\hat{H}-E_{r}\right| \tilde{\chi}_{q}^{a}\right\rangle\right|^{2}
$$

where $\mathbf{T}$ is the matrix obtained after diagonalization of the matrix of $2 \mathrm{~h}$-configurations, and $\mathbf{d}^{a}$ is the matrix of expansion coefficients of the Fano-CI states. The matrix elements $\left\langle\Phi\left|\hat{H}-E_{r}\right| \tilde{\chi}_{q}^{a}\right\rangle$ are easily evaluated as sums of two-electron integrals and HF orbital energies $[13]$.

\section{B. First-order perturbation theory based model}

In [11], we have shown that superexchange ICD mechanism in NeHeNe is mediated by $\mathrm{Ne}^{+} \mathrm{He}^{-} \mathrm{Ne}^{+}$virtual states. In that case, we have derived an approximate expression for the ICD widths as a function of the NeNe internuclear distance. In order to interpret the results 
from the Fano-CI calculations we employ the same model here. A more detailed derivation is however given. Furthermore, we use the symmetries of the system to analyze the partial ICD widths. We start from Eq. 1 for a given channel $\beta$

$$
\Gamma_{\beta}=2 \pi\left|\left\langle\Phi\left|\hat{H}-E_{r}\right| \chi_{\beta, \epsilon_{\beta}}\right\rangle\right|^{2}
$$

Using first-order perturbation theory and a single configuration state function (CSF) for each state, we have

$$
|\Phi\rangle=c_{i}\left|\Psi_{0}\right\rangle
$$

and

$$
\left|\chi_{\beta, \epsilon_{\beta}}\right\rangle=c_{a}^{\dagger} c_{j} c_{k}\left|\Psi_{0}\right\rangle+\sum_{J} \frac{H_{J f}^{\beta}}{E_{f}^{\beta}-E_{J}} c_{J}^{\dagger} c_{j} c_{k}\left|\Psi_{0}\right\rangle
$$

where $i, j$ and $k$ are occupied orbitals of the donor and acceptor systems defining the channel $\beta, a$ represents a continuum orbital and $J$ is a virtual orbital of the bridge atom. The creation and annhilation operators are denoted as $c^{\dagger}$ and $c$, respectively. In the above equation, $H_{J f}$ is the Hamiltonian matrix element between the $c_{a}^{\dagger} c_{j} c_{k}\left|\Psi_{0}\right\rangle$ and $c_{J}^{\dagger} c_{j} c_{k}\left|\Psi_{0}\right\rangle$ CSFs, where $\left|\Psi_{0}\right\rangle$ denotes the Hartree-Fock ground state. The energy expectation values of these two CSFs are $E_{f}^{\beta}$ and $E_{J}$, respectively.

Including Eqs. 6 and 7 into Eq. 5, we obtain

$$
\left\langle\Phi\left|\hat{H}-E_{r}\right| \chi_{\beta, \epsilon_{\beta}}\right\rangle=\sqrt{\frac{3}{2}}\left(\langle a i \mid j k\rangle-\langle a i \mid k j\rangle+\sum_{J} \frac{H_{J f}^{\beta}}{E_{f}^{\beta}-E_{J}}(\langle J i \mid j k\rangle-\langle J i \mid k j\rangle)\right)
$$

In the above expression, we assume that the final state $\beta$ is a triplet state. Similar derivation is easily done for a singlet state [13]. The first two terms on the r.h.s. of Eq. 8 correspond to the direct ICD process (the first term is the direct term and the second the exchange one as in [22]). The terms in the sum over $J$ are the ones corresponding to the superexchange mechanism. In what follows, we focus on the range of distances where these terms dominate over the direct ICD ones (e.g. $\mathrm{R}<6-7 \AA$ for linear NeHeNe trimer, see [11] and below). Furthermore, we assume that i) the donor and acceptor orbitals form an orthogonal basis set and ii) the donor and acceptor orbitals are NOT orthogonal to those of the bridge atom. We then expand the Coulomb operator as in [22]. In the particular case of a linear geometry and keeping only the dominant terms, Eq. 8 then reads

$$
\left\langle\Phi\left|\hat{H}-E_{r}\right| \chi_{\beta, \epsilon_{\beta}}\right\rangle=\sqrt{\frac{3}{2}} \sum_{J} \frac{H_{J f}^{\beta}}{R^{2}\left(E_{f}^{\beta}-E_{J}\right)}(\langle J \mid j\rangle\langle i|z| k\rangle-\langle J \mid k\rangle\langle i|z| j\rangle)
$$


As defined above, $\mathrm{R}$ is the distance between the donor and acceptor species (i.e. Ne-Ne and Ne-Ar below). Several observations can be made at this point. First, on contrary to the direct ICD mechanism whose rates are proportional to $\mathrm{R}^{-6}$, the superexchange terms exhibit a $\mathrm{R}^{-4}$ behavior. Note, however, that the terms $\langle J \mid k\rangle$ and $\langle J \mid j\rangle$ also depend on $\mathrm{R}$. In [11], we assumed an exponential behavior for these terms. Second, the superexchange mechanism can only be operative if the states of the bridge atom lie close in energy to the resonance since the couplings are inversely proportional to the energy difference. Finally, owing to electric dipole selection rules, only the channels with proper symmetries are enhanced in the presence of the bridge atom compared to the isolated case, i.e. there is no superexchange contribution if $\langle i|z| l\rangle$ and $\langle i|z| k\rangle$ are zero for symmetry reasons. For example, in the case of NeHeNe trimer after $2 \mathrm{~s} \sigma_{g}$ ionization only ICD channels leading to $\mathrm{Ne}^{+} \mathrm{HeNe}^{+}$with at least one hole in $2 p \sigma_{u}$ are enhanced.

The approximate expression obtained in Eq. 9 is used in the following to discuss the Fano-CI results.

\section{RESULTS AND DISCUSSION}

\section{A. Superexchange ICD in neon-helium-neon trimer}

We first discuss the total ICD widths for linear NeHeNe trimer obtained with the FanoCI method. Note that in [11] these widths were computed with the Fano-ADC method. Similar results are obtained here (see [13] for a comparison between the two methods). Fig. 1 shows the total ICD widths for ${ }^{2} \Sigma_{g}\left(2 s^{-1}\right)$ (left panel) and ${ }^{2} \Sigma_{u}\left(2 s^{-1}\right)$ (right panel) states in NeHeNe compared to NeNe. We assume a linear geometry with helium located at the center of mass of the neon dimer. As in [11], a significant enhancement of the ICD widths is observed in the presence of the helium atom for $\mathrm{R}$ smaller than 6-7 $\mathrm{A}$.

After ICD, the doubly-ionized trimer can be in one of the following twelve states (labeled as in the case of the isolated neon dimer): ${ }^{1,3} \Delta_{g},{ }^{1,3} \Pi_{g},{ }^{1,3} \Pi_{u},{ }^{1} \Sigma_{g}^{+}(\mathrm{x} 2),{ }^{1} \Sigma_{u}^{-},{ }^{3} \Sigma_{u}^{+}(\mathrm{x} 2)$ and ${ }^{3} \Sigma_{g}^{-}$. In Figs. 2 and 3, we report the partial widths of the states that are enhanced by the superexchange ICD mechanism. As seen in the figures, only ICD channels leading to ${ }^{1,3} \Pi_{g}$,

${ }^{1,3} \Pi_{u}$, one of the ${ }^{1} \Sigma_{g}^{+}$and ${ }^{3} \Sigma_{u}^{+}$states have enhanced widths in the presence of the bridge atom compared to the isolated case. This is in agreement with the symmetry reasoning 

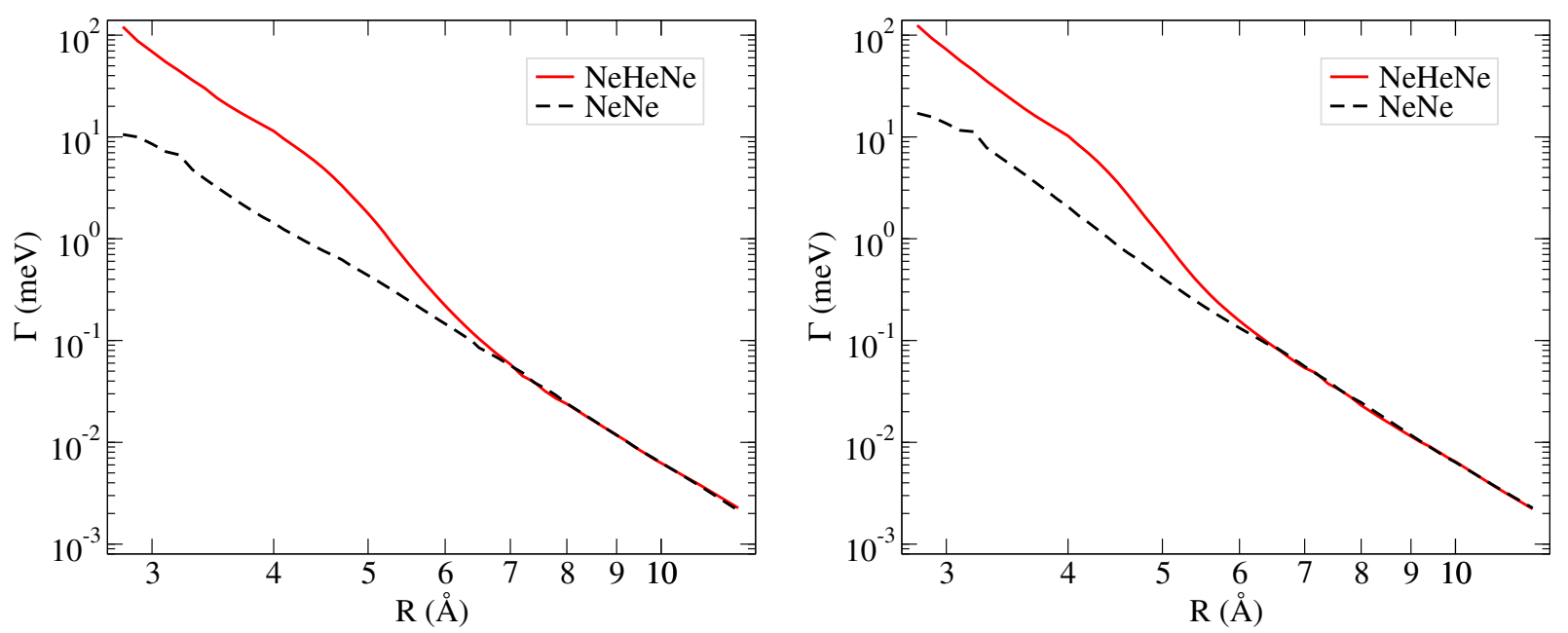

FIG. 1: Comparison between the total decay widths of the $\mathrm{Ne}^{+}\left(2 \mathrm{~s}^{-1}\right){ }^{2} \Sigma_{g, u}^{+}$states in $\mathrm{Ne}_{2}$ (black dashed lines) and NeHeNe (red full lines). Left panel: $\mathrm{Ne}^{+}\left(2 \mathrm{~s}^{-1}\right)^{2} \Sigma_{g}^{+}$. Right panel:

$$
\mathrm{Ne}^{+}\left(2 \mathrm{~s}^{-1}\right)^{2} \Sigma_{u}^{+} \text {. }
$$

obtained with the model presented in Sec. II B.

We note that for some distances a few partial ICD widths are smaller in the presence of the bridge atom than that in the isolated case. Such a decrease can be due to interference effects between the direct ICD and the superexchange ICD pathways, as well as some screening effects of the bridge species reducing thus the interaction between the two neon atoms. Further works are needed to investigate these effects.

\section{B. Superexchange ICD in neon-helium-argon trimer}

We now discuss the ICD process in linear NeHeAr trimer. For the superexchange ICD mechanism, the main difference in this system compared to $\mathrm{NeHeNe}$ is the energies of the bridge states relative to the resonance energy. Assuming the energies of the bridge states are equal to those of $\mathrm{He}^{-}$with two point charges at the position of the donor and acceptor atoms and that the energy of 2s-ionized neon donor atom does not depend significantly on the nature of the acceptor atom, the difference between NeHeNe and NeHeAr is the ionization potential difference of $\mathrm{Ne}$ and $\mathrm{Ar}$, which is about $-5 \mathrm{eV}$. The bridge states lie therefore further away from the resonance energy in the case of NeHeAr. Assuming similar $H_{J f}$, dipole and overlap terms in Eq. 9, it is expected that the superexchange mechanism is 

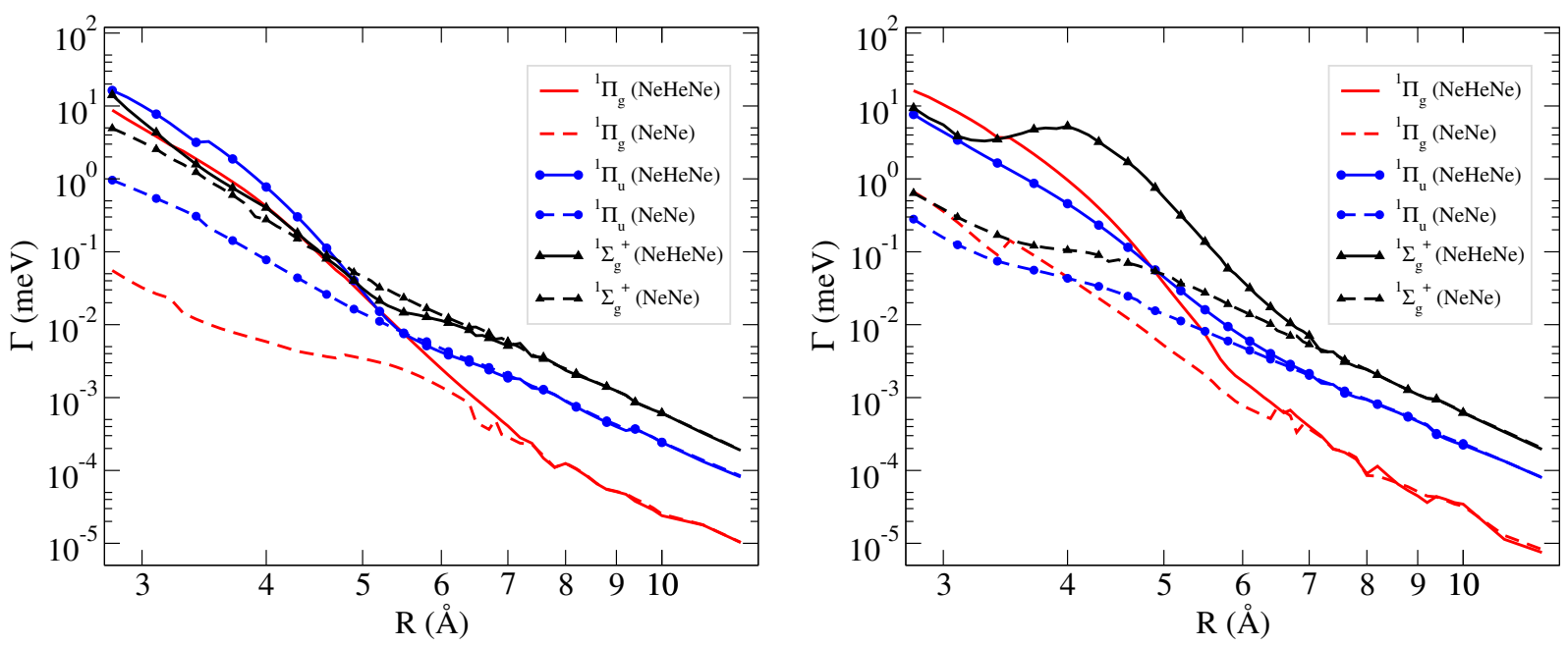

FIG. 2: Comparison between the partial decay widths of the $\mathrm{Ne}^{+}\left(2 \mathrm{~s}^{-1}\right)^{2} \Sigma_{g, u}^{+}$states in $\mathrm{Ne}_{2}$ (dashed lines) and NeHeNe (full lines) to singlet final states. We show only the channels, whose decay widths are enhanced as a result of the superexchange ICD $-{ }^{1} \Pi_{g},{ }^{1} \Pi_{u},{ }^{1} \Sigma_{g}^{+}$.

Left panel: $\mathrm{Ne}^{+}\left(2 \mathrm{~s}^{-1}\right)^{2} \Sigma_{g}^{+}$. Right panel: $\mathrm{Ne}^{+}\left(2 \mathrm{~s}^{-1}\right)^{2} \Sigma_{u}^{+}$.
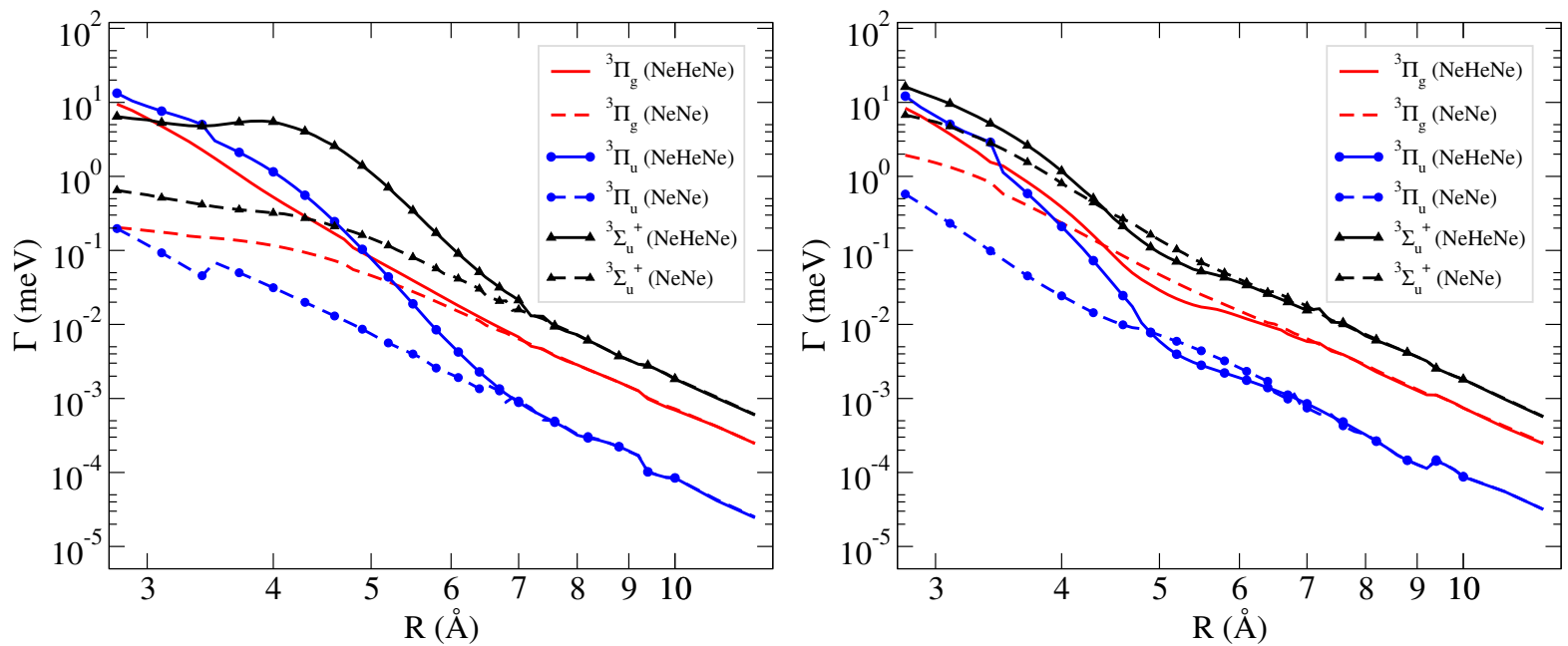

FIG. 3: Comparison between the partial decay widths of the $\mathrm{Ne}^{+}\left(2 \mathrm{~s}^{-1}\right)^{2} \Sigma_{g, u}^{+}$states in $\mathrm{Ne}_{2}$ (dashed lines) and NeHeNe (full lines) to triplet final states. We show only the channels, whose decay widths are enhanced as a result of the superexchange ICD $-{ }^{3} \Pi_{g},{ }^{3} \Pi_{u},{ }^{3} \Sigma_{u}^{+}$. Left panel: $\mathrm{Ne}^{+}\left(2 \mathrm{~s}^{-1}\right)^{2} \Sigma_{g}^{+}$. Right panel: $\mathrm{Ne}^{+}\left(2 \mathrm{~s}^{-1}\right)^{2} \Sigma_{u}^{+}$. 


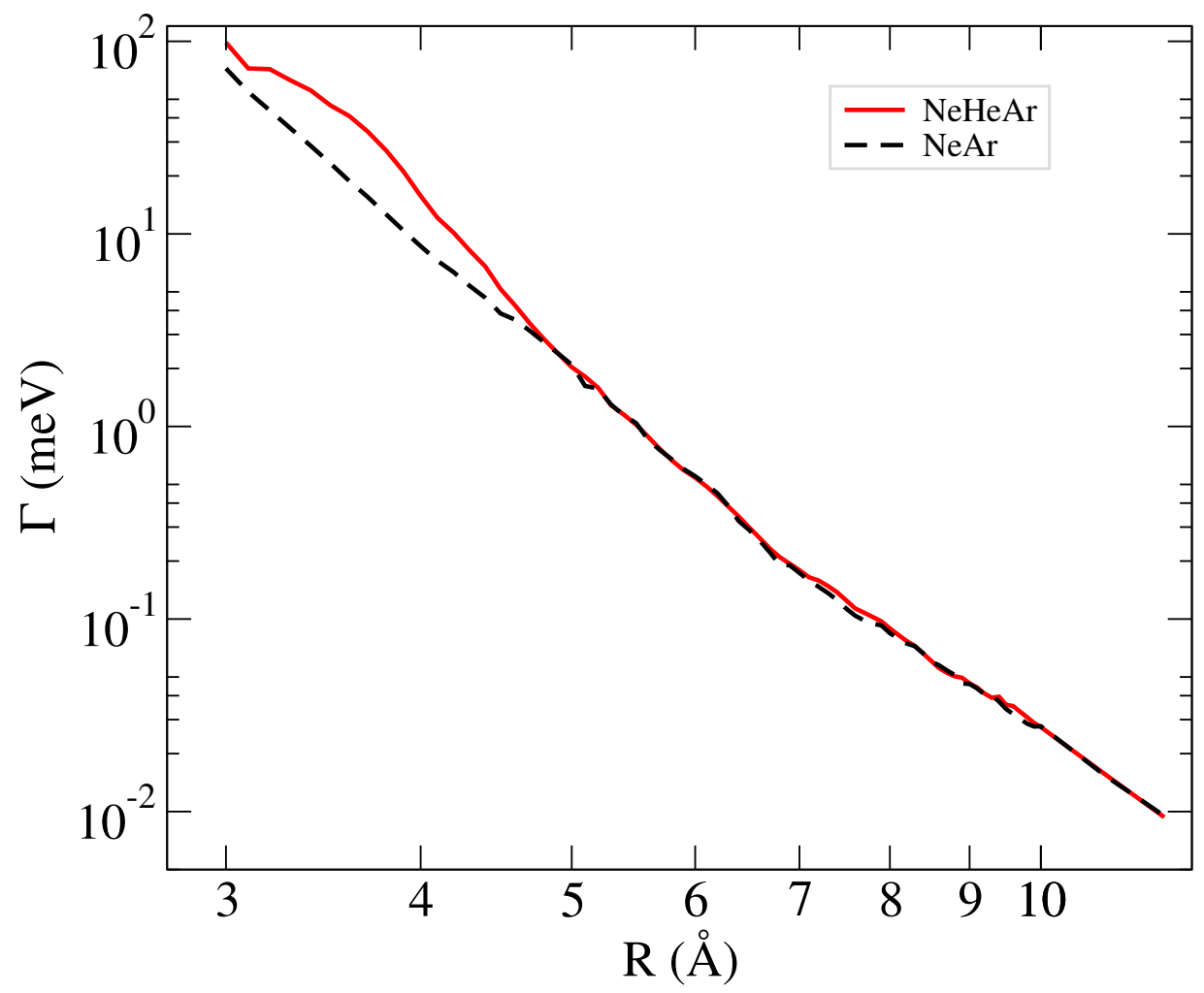

FIG. 4: Comparison between the total decay widths of the $\mathrm{Ne}^{+}\left(2 \mathrm{~s}^{-1}\right)^{2} \Sigma^{+}$states in NeAr (black dashed line) and NeHeAr (red full line).
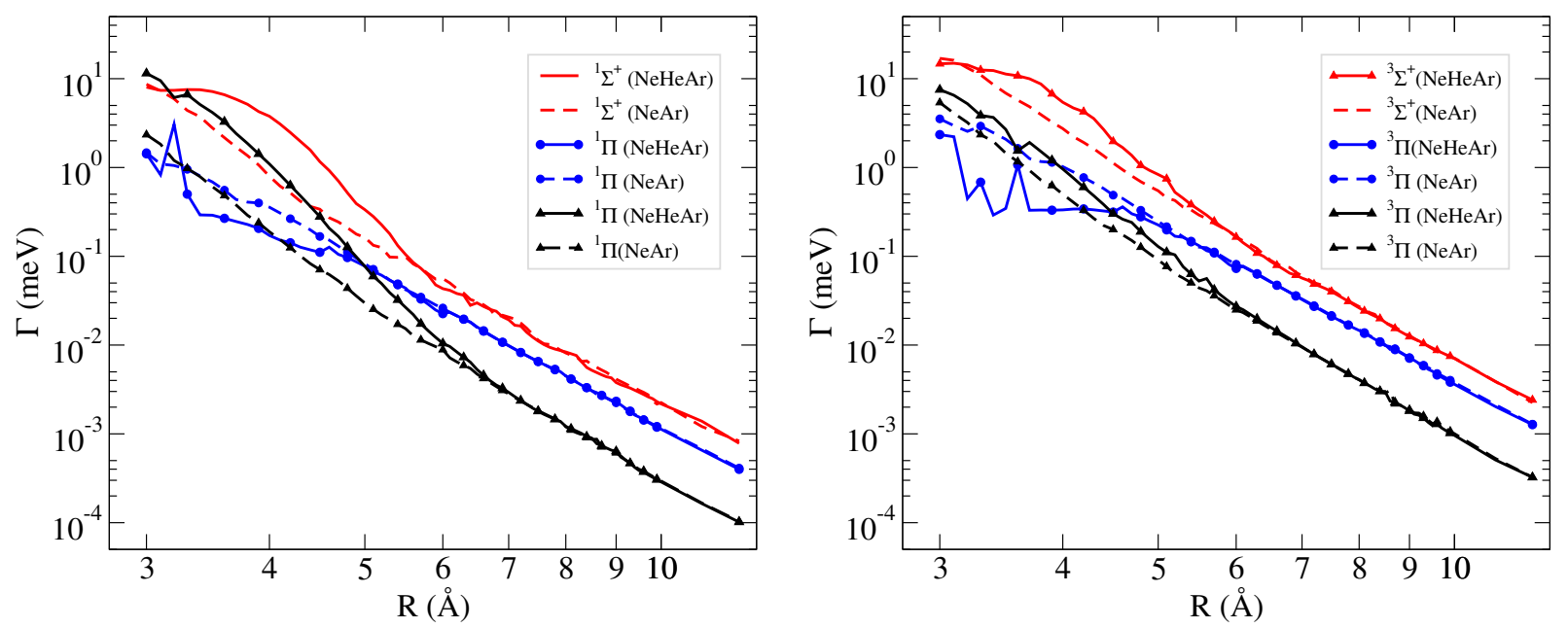

FIG. 5: Comparison between the partial decay widths of the $\mathrm{Ne}^{+}\left(2 s^{-1}\right)^{2} \Sigma^{+}$state in $\mathrm{NeAr}$ and NeHeAr to singlet (left panel) and triplet (right panel) final states. We show only the channels, whose decay widths are enhanced as a result of the superexchange ICD $-{ }^{1} \Pi$,

$$
{ }^{1} \Sigma^{+} \text {and }{ }^{3} \Pi,{ }^{3} \Sigma^{+} .
$$


less effective in NeHeAr than in NeHeNe. In [13], we showed that the virtual states of the bridge atom are between $3 \mathrm{eV}$ and $16 \mathrm{eV}$ below the resonance energy in the case of NeHeNe. A shift of $-5 \mathrm{eV}$ in Eq. 9 leads to a decrease of the superexchange ICD terms by a factor of between 0.35 and 0.6 .

The total ICD widths in NeHeAr are shown in Fig. 4. As expected, the enhancement of the ICD widths in the presence of the helium atom is weaker and, moreover, starts to be operative at shorter interatomic distances in the case of NeHeAr compared to NeHeNe. Indeed, the superexchange mechanism is seen at distances below $5 \AA$ in NeHeAr while it starts below $7 \AA$ for NeHeNe. Furthermore, the maximum enhancement is only of a factor of about 2 for the NeHeAr and of about 7 for NeHeNe. This decrease in the efficiency of the superexchange ICD mechanism in NeHeAr seen in the ab initio results agrees quantitatively with that predicted by our first-order perturbation theory based model.

For completeness, we show the partial ICD widths in NeHeAr in Fig. 5. Among the final states, which are ${ }^{1,3} \Delta,{ }^{1,3} \Pi(\mathrm{x} 2),{ }^{1,3} \Sigma^{+}(\mathrm{x} 2)$ and ${ }^{1,3} \Sigma^{-}$, only one of each ${ }^{1,3} \Pi$ and ${ }^{1,3} \Sigma^{+}$are enhanced in agreement with the pertubation-theory based model. Note that in the trimer case, the partial ICD widths for the $\Pi$ states exhibit sharp peaks for some distances (e.g. at $\mathrm{R}=3.2 \AA$ in the left panel of Fig. 5) which are attributed to interferences between the two adiabatic $\Pi$ states in each spin symmetry. Such interference effects will be discussed in a future publication.

\section{CONCLUSIONS}

We have analyzed in detail the superexchange ICD mechanism in NeHeNe and NeHeAr. Total and partial ICD widths, computed with the Fano-CI method, are reported. It is shown that, owing to symmetry reasons, only some ICD channels are enhanced in the presence of helium as a bridge atom compared to isolated NeNe and NeAr dimers. Furthermore, due to the lower ionization potential of argon compared to neon, the energy of the bridge states of NeHeAr are further away from the resonance energy compared to NeHeNe. The superexchange contributions are therefore smaller and appear at shorter interatomic distances. A simple model based on first-order perturbation theory and a Hartree-Fock-like description of the states is reported. This model explains the observation made from the Fano-CI results and thus provides insight into the superexchange ICD mechanism. NeHeNe 
and NeHeAr have been employed in this work because they allow an accurate theoretical description. However, related phenomena are known in resonance energy transfer within molecular aggregates (see for example [23]) and excitation transfer along covalent bonds in large molecules [24], which suggest that superexchange ICD mechanism is more general. Further experimental and theoretical works on atomic and molecular clusters with different bridge systems are needed to unravel the full importance of this new mechanism.

\section{ACKNOWLEDGMENTS}

This project has received funding from the Research Executive Agency (REA) under the European Union's Horizon 2020 research and innovation programme Grant agreement No. 705515. P. K. and P. V. acknowledge financial support by the Czech Science Foundation (Project GAČR No. 17-10866S).

[1] L. S. Cederbaum, J. Zobeley, and F. Tarantelli, Phys. Rev. Lett. 79, 4778 (1997).

[2] S. Marburger, O. Kugeler, U. Hergenhahn, and T. Möller, Phys. Rev. Lett. 90, 203401 (2003).

[3] T. Jahnke, A. Czasch, M. S. Schöffler, S. Schössler, A. Knapp, M. Käsz, J. Titze, C. Wimmer, K. Kreidi, R. E. Grisenti, A. Staudte, O. Jagutzki, U. Hergenhahn, H. Schmidt-Böcking, and R. Dörner, Phys. Rev. Lett. 93, 163401 (2004).

[4] R. Santra, J. Zobeley, L. S. Cederbaum, and N. Moiseyev, Phys. Rev. Lett. 85, 4490 (2000).

[5] K. Schnorr, A. Senftleben, M. Kurka, A. Rudenko, L. Foucar, G. Schmid, A. Broska, T. Pfeifer, K. Meyer, D. Anielski, R. Boll, D. Rolles, M. Kübel, M. F. Kling, Y. H. Jiang, S. Mondal, T. Tachibana, K. Ueda, T. Marchenko, M. Simon, G. Brenner, R. Treusch, S. Scheit, V. Averbukh, J. Ullrich, C. D. Schröter, and R. Moshammer, Phys. Rev. Lett. 111, 093402 (2013).

[6] V. Averbukh, P. V. Demekhin, P. Kolorenč, S. Scheit, S. D. Stoychev, A. I. Kuleff, Y.-C. Chiang, K. Gokhberg, S. Kopelke, N. Sisourat, and L. S. Cederbaum, J. Electron Spectrosc. Relat. Phenom. 183, 36 (2011).

[7] U. Hergenhahn, J. Electron Spectrosc. Relat. Phenom. 184, 78 (2011).

[8] T. Jahnke, J. Phys. B: At. Mol. Opt. Phys. 48, 082001 (2015). 
[9] See http://www.pci.uni-heidelberg.de/tc/usr/icd/ICD.refbase.html for the complete list of ICD papers.

[10] V. Averbukh, I. B. Müller, and L. S. Cederbaum, Phys. Rev. Lett. 93, 263002 (2004).

[11] T. Miteva, S. Kazandjian, P. Kolorenč, P. Votavová, and N. Sisourat, Phys. Rev. Lett. 119, 083403 (2017).

[12] R. Bennett, P. Votavová, P. Kolorenč, T. Miteva, N. Sisourat, and S. Y. Buhmann, Phys. Rev. Lett. 122, 153401 (2019).

[13] T. Miteva, S. Kazandjian, and N. Sisourat, Chem. Phys. 482, 208 (2017), Electrons and nuclei in motion - correlation and dynamics in molecules (on the occasion of the 70th birthday of Lorenz S. Cederbaum).

[14] U. Fano, Phys. Rev. 124, 1866 (1961).

[15] G. Howat, T. Åberg, and O. Goscinski, J. Phys. B At. Mol. Opt. Phys. 11, 1575 (1978).

[16] A. U. Hazi, in Electron-Molecule and Photon-Molecule Collisions, edited by T. Rescigno, V. McKoy, and B. Schneider (Plenum Press, New York and London, 1979).

[17] P. W. Langhoff, in Electron-Molecule and Photon-Molecule Collisions, edited by T. Rescigno, V. McKoy, and B. Schneider (Plenum Press, New York and London, 1979).

[18] F. Müller-Plathe and G. H. F. Diercksen, Phys. Rev. A 40, 696 (1989).

[19] V. Averbukh and L. S. Cederbaum, J. Chem. Phys. 123, 204107 (2005).

[20] T. H. Dunning, J. Chem. Phys. 90, 1007 (1989).

[21] K. Kaufmann, W. Baumeister, and M. Jungen, J. Phys. B At. Mol. Opt. Phys. 22, 2223 (1989).

[22] R. Santra and L. S. Cederbaum, Phys. Rep. 368, 1 (2002).

[23] A. Salam, J. Chem. Phys. 136, 014509 (2012).

[24] A. I. Kuleff, Chem. Phys. 482, 216 (2017). 